\title{
Dependence of Ga-doped $\mathrm{ZnO}$ thin film properties on different sputtering process parameters: Substrate temperature, sputtering pressure and bias voltage
}

\author{
M.V. Castro, C.J. Tavares * \\ Centre of Physics, University of Minho, 4710-057 Braga, Portugal
}

\section{A R T I C L E I N F O}

\section{Article history}

Received 25 July 2014

Received in revised form 10 April 2015

Accepted 14 April 2015

Available online 22 April 2015

\section{Keywords:}

Thin films

Transparent conducting oxide

Gallium-doped zinc oxide

Sputtering

Electrical properties

Pressure

Bias voltage

Substrate temperature

\begin{abstract}
A B S T R A C T
This paper reports on the effects of different sputtering deposition process parameters (substrate temperature, sputtering pressure and bias voltage) on the electrical, optical, structural and morphological properties of gallium-doped $\mathrm{ZnO}$ ( $\mathrm{ZnO}: \mathrm{Ga}$ ) of $\sim 1 \mu \mathrm{m}$ thick. These highly transparent and conductive films were deposited on glass surfaces by d.c. pulsed magnetron sputtering from a $\mathrm{GZO}\left(\mathrm{ZnO}(95.5): \mathrm{Ga}_{2} \mathrm{O}_{3}(4.5)\right)$ ceramic target in an argon atmosphere. X-ray diffraction experiments show that all films have a hexagonal wurtzite structure with the [001] preferred crystallographic direction, and the morphology of the films (obtained from scanning electron microscope analysis) is sensitive to the process parameters. All ZnO:Ga films have an average transmittance above $80 \%$ in the visible region, and the lowest electrical resistivity of $3.03 \times 10^{-4} \Omega \cdot \mathrm{cm}$ was achieved for the sample submitted to the lowest bias voltage $(-40 \mathrm{~V})$, which corresponds to a carrier concentration and a carrier mobility of $6.99 \times 10^{20} \mathrm{~cm}^{-3}$ and $29.49 \mathrm{~cm}^{2} \mathrm{~V}^{-1} \mathrm{~s}^{-1}$, respectively. A high substrate temperature, high sputtering pressure and low negative bias voltage (within the range of studied parameters) proved to be very promising on obtaining optimized $\mathrm{ZnO}$ :Ga films, ensuring suitable properties for application as transparent electrodes in photovoltaic cells.
\end{abstract}

C 2015 Elsevier B.V. All rights reserved.

\section{Introduction}

Transparent conductive materials are simultaneously optically transparent and electrically conductive [1]. Among the different transparent conductive oxide (TCO) materials, zinc oxide $(\mathrm{ZnO})$ is a promising alternative to indium tin oxide (ITO) in TCO applications, due to its low cost and relatively low deposition temperature and stability in hydrogen plasma, compared to ITO and $\mathrm{SnO}_{2}$. Furthermore, $\mathrm{ZnO}$ is an abundant and nontoxic material [2-4]. Undoped $\mathrm{ZnO}$ films with a hexagonal wurtzite structure generally exhibits n-type conduction due to nonstoichiometry, presenting high electrical resistivity due to a low carrier concentration (with a typical carrier concentration of $\sim 10^{17} / \mathrm{cm}^{3}$ ) $[2,3,5,6]$. Therefore, it is common to use $\mathrm{Al}$, In or Ga to improve the electrical properties of $\mathrm{ZnO}$ [3]. It has been reported that gallium ( $\mathrm{Ga}$ ) is less reactive and more resistive to oxidation when compared to aluminum (Al) $[3,5,7]$. Besides that, the ionic radius of $\mathrm{Ga}^{3+}$ is smaller than that of $\mathrm{Zn}^{2+}$, but larger than that of $\mathrm{Al}^{3+}$, so $\mathrm{Ga}^{3+}$ ions fit into $\mathrm{ZnO}$ lattice more easily than $\mathrm{Al}$. The covalent bond lengths of $\mathrm{Ga}-\mathrm{O}$ and $\mathrm{Zn}-\mathrm{O}$ are estimated to be 1.92 and $1.97 \AA$, respectively. The slightly smaller bond length of $\mathrm{Ga}-\mathrm{O}$ than that of $\mathrm{Zn}-\mathrm{O}$ is an advantage since it should result in less deformation of $\mathrm{ZnO}$ lattice, even when using high gallium

\footnotetext{
* Corresponding author. Tel.: + 351253510474.

E-mail address: ctavares@fisica.uminho.pt (C.J. Tavares).
}

concentrations $[2,3,5,7,8]$. The choice of gallium as dopant, in the present paper, was based on these advantages.

Among the available techniques for deposition of $\mathrm{ZnO}$ thin films, magnetron sputtering has been the most preferred method due to its advantages, such as: low substrate temperature, good reproducible surface morphology, good adhesion of the film on substrate and long-term stability $[2,8]$. In this work, highly transparent and conductive galliumdoped $\mathrm{ZnO}(\mathrm{ZnO}: \mathrm{Ga}$ ) films were grown by d.c. pulsed magnetron sputtering. The influence of different process parameters, such as: substrate temperature, sputtering pressure and bias voltage, on the electrical, optical and structural properties of the as-deposited $\mathrm{ZnO}$ :Ga thin films, was investigated.

\section{Materials and methods}

\subsection{Thin film deposition}

ZnO:Ga transparent and conductive films were deposited in an argon (Ar) atmosphere on glass substrates $(76 \mathrm{~mm} \times 26 \mathrm{~mm}$ and $1 \mathrm{~mm}$ thick), using a custom-built magnetron sputtering equipment. A GZO ceramic target ( $\mathrm{ZnO}(95.5): \mathrm{Ga}_{2} \mathrm{O}_{3}(4.5)$ ) was used, with a diameter of $10 \mathrm{~cm}$, fabricated by FHR Anlagenbau $\mathrm{GmbH}$. Prior to the depositions the chamber was evacuated using a turbo molecular pump to ensure a base pressure of $\sim 10^{-4} \mathrm{~Pa}$. 
The glass substrates were ultrasonically cleaned in isopropyl alcohol (2-propanol) and rinsed in an ultrasonic bath for $15 \mathrm{~min}$, in order to reduce the contaminants on its surface that may arise during storage and handling, for example, which moreover deteriorate the adhesion between substrate and film [9].

$\mathrm{ZnO}$ :Ga films depositions were carried out in static mode, with the following fixed parameters: cathode current density of $5.1 \mathrm{~mA} / \mathrm{cm}^{2}$, d.c. pulsed frequency of $140 \mathrm{kHz}$ and $65 \%$ duty-cycle, bipolar d.c. pulsed bias with a frequency of $100 \mathrm{kHz}, 70 \%$ duty-cycle with a positive pulse of $16 \mathrm{~V}$ on the reverse cycle (MAC Co. power supplies). The deposition temperature was monitored directly by placing a K-type thermocouple (Omega Engineering Limited) on the surface of a dummy sample for all runs. The influence of three deposition parameters (substrate temperature, sputtering pressure and substrate bias voltage), on the final properties of the as-deposited $\mathrm{ZnO}$ :Ga thin films, was studied. Therefore, three series of depositions were performed: substrate temperature effect (series 1); sputtering pressure effect (series 2); and study of substrate bias voltage effect (series 3 ). The variable process parameters, used in each deposition series, are presented in Table 1.

\subsection{Electrical characterization}

In order to obtain the electrical properties, such as: conductivitytype, electrical resistivity $(\rho)$, electrical mobility $(\mu)$ and carrier concentration ( $n$ ), of ZnO:Ga thin films, Hall effect measurements with a magnetic field of $0.560 \mathrm{~T}$ were carried out at room temperature, under atmospheric pressure. A Hall effect measurement system - Ecopia model HMS-5000 - with d.c. four-point probe in the Van der Pauw configuration was used for this purpose.

The Hall effect measurement is a reliable and direct method for assessing the electrical properties of semiconductor materials. Carrier concentration and carrier mobility were obtained from [10]:

$n=8 \times 10^{-8} \frac{I B}{d e\left|V_{s}\right|}$

$\mu=\frac{1}{e n d R_{S}}$

In the above equations, $I$ is the electric current, $B$ is the magnetic field (in Gauss), $d$ is the film thickness, eis the elementary charge, $V_{S}$ is the total Hall voltage and $R_{S}$ is the sheet resistance. For a good accuracy of the results, the contacts should be sufficiently small and at the periphery of the sample. Furthermore, the thin film should be of uniform thickness and free of pinholes [10].

\subsection{Optical, structural and morphological characterization}

Optical transmittance spectra were obtained using a Shimadzu UV2501PC spectrophotometer (in a range of wavelengths between 300 and $900 \mathrm{~nm}$ ), using air as reference.

The crystallographic structure of the ZnO:Ga films was investigated from X-ray diffraction (XRD) analyses, using a Bruker AXS D8 Discover diffractometer, operated in $\theta-2 \theta$ geometry with $C u K \alpha$ radiation. XRD patterns were acquired with a step size of $0.04^{\circ}$ and an integration time of $1.5 \mathrm{~s}$. The Topas software from Bruker AXS was used to refine the diffraction patterns and hence obtain the structural data such as lattice parameters and crystallite grain size, among other parameters.

The morphology and cross-section of the obtained $\mathrm{ZnO}$ :Ga films were investigated by scanning electron microscopy (SEM) with a FEI NOVA 200 FEG-SEM equipment operating at a maximum voltage of $30 \mathrm{kV}$.

\section{Results and discussion}

For the application of $\mathrm{ZnO}: \mathrm{Ga}$ thin films as a transparent conductor electrodes in photovoltaic applications, the most important factors to take into account are the optical transmittance (which must be high) and electrical resistivity (which should be as low as possible) [11]. In the present paper, it is presented the study of the influence of different sputtering process parameters: substrate temperature, sputtering pressure and substrate bias voltage, on the electrical, optical and structural properties of $\mathrm{ZnO}$ :Ga thin films.

\subsection{Effect of substrate temperature}

Fig. 1 shows the $\rho, n$ and $\mu$ as a function of substrate temperature $\left(T_{s}\right)$ during ZnO:Ga film deposition (series 1 in Table 1 ). The electrical resistivity decreases sharply from $6.61 \times 10^{-4} \Omega \cdot \mathrm{cm}$ to $3.71 \times 10^{-4} \Omega \cdot \mathrm{cm}$ as the substrate temperature increases from 270 to $320^{\circ} \mathrm{C}$. These values of $\rho$ are very promising for $\mathrm{ZnO}$ :Ga application as transparent conductive oxide electrodes [12,13]. With increasing substrate temperature, more $\mathrm{Ga}$ atoms are activated into the $\mathrm{ZnO}$ lattice as donors - there is an increase in diffusion of $\mathrm{Ga}$ atoms from interstitial locations and grain boundaries into the $\mathrm{Zn}$ cation sites -, which leads to the observed successive increase in carrier concentration, since the Ga atom is trivalent and $\mathrm{Zn}$ has a valence of 2 [14-16]. A similar behavior was reported by other authors $[14,17]$. In studies that involved the effect of the substrate temperature on the properties of Ga-doped $\mathrm{ZnO}$ films, Park and coworkers [13] have achieved an electrical resistivity of $5.3 \times 10^{-4} \Omega \cdot \mathrm{cm}$ using a substrate temperature of $225^{\circ} \mathrm{C}$; also S. Wang and co-workers [14] have achieved a minimum electrical resistivity of $5.8 \times 10^{-4} \Omega \cdot \mathrm{cm}$ using a substrate temperature of $350{ }^{\circ} \mathrm{C}$, which are slightly higher

Table 1

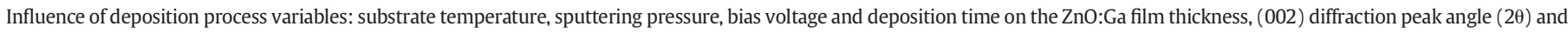
respective crystalline grain size. The thickness $(d)$ was evaluated from the cross-section analyses on the SEM micrographs.

\begin{tabular}{|c|c|c|c|c|c|c|c|}
\hline \multirow[b]{2}{*}{ Series } & \multicolumn{4}{|l|}{ Process variable } & \multirow[b]{2}{*}{$\begin{array}{l}\text { Film thickness, } d \\
(\mu \mathrm{m})\end{array}$} & \multirow[b]{2}{*}{$\begin{array}{l}(002) \text { diffraction peak, } 2 \theta \\
\left({ }^{\circ}\right)\end{array}$} & \multirow[b]{2}{*}{$\begin{array}{l}\text { Grain size } \\
(\mathrm{nm})\end{array}$} \\
\hline & $\begin{array}{l}\text { Substrate temperature } \\
\left({ }^{\circ} \mathrm{C}\right)\end{array}$ & $\begin{array}{l}\text { Sputtering pressure } \\
(\mathrm{Pa})\end{array}$ & $\begin{array}{l}\text { Bias voltage } \\
(\mathrm{V})\end{array}$ & $\begin{array}{l}\text { Deposition time } \\
\text { (min) }\end{array}$ & & & \\
\hline \multirow[t]{4}{*}{1} & 270 & 0.27 & -60 & 15 & $0.94 \pm 0.01$ & 34.20 & 22.6 \\
\hline & 290 & & & & $1.02 \pm 0.01$ & 34.20 & 35.1 \\
\hline & 310 & & & & $1.06 \pm 0.01$ & 34.20 & 38.0 \\
\hline & 320 & & & & $1.25 \pm 0.01$ & 34.20 & 42.3 \\
\hline \multirow[t]{4}{*}{2} & 300 & 0.30 & -60 & 12.5 & $0.83 \pm 0.01$ & 34.20 & 13.1 \\
\hline & & 0.38 & & & $0.86 \pm 0.01$ & 34.20 & 31.4 \\
\hline & & 0.40 & & & $0.87 \pm 0.01$ & 34.24 & 38.0 \\
\hline & & 0.42 & & & $0.91 \pm 0.01$ & 34.28 & 53.0 \\
\hline \multirow[t]{5}{*}{3} & 320 & 0.27 & 0 & 12.5 & $1.29 \pm 0.04$ & 34.24 & 55.7 \\
\hline & & & -40 & & $1.40 \pm 0.01$ & 34.24 & 74.3 \\
\hline & & & -60 & & $1.14 \pm 0.01$ & 34.20 & 41.8 \\
\hline & & & -90 & & $0.89 \pm 0.01$ & 34.12 & 35.5 \\
\hline & & & -120 & & $0.87 \pm 0.01$ & 34.12 & 12.7 \\
\hline
\end{tabular}




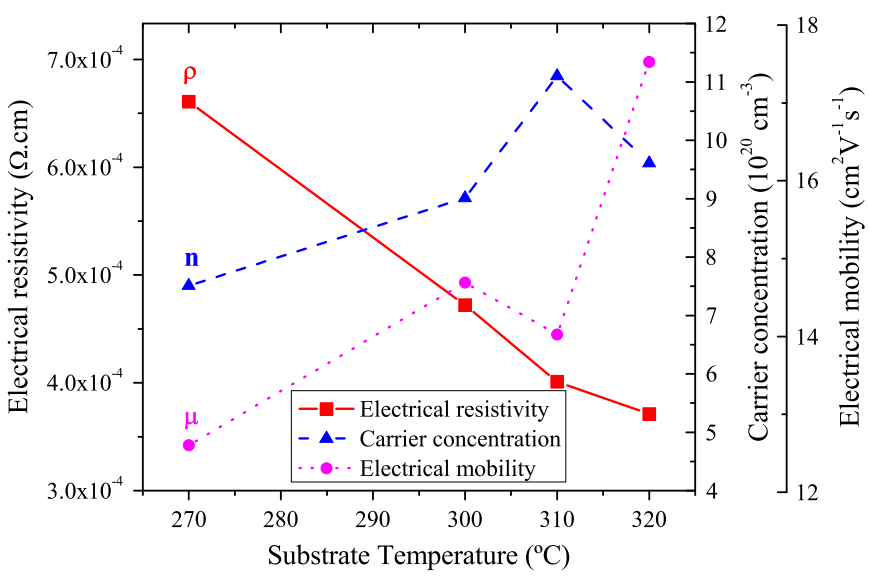

Fig. 1. Effect of substrate temperature on the electrical properties of $\mathrm{ZnO}$ :Ga thin films, for deposition conditions of series 1 in Table 1.

values than that obtained in the present manuscript for a substrate temperature of $320^{\circ} \mathrm{C}$. The changes in both carrier concentration (from $7.51 \times 10^{20}$ to $9.61 \times 10^{20} \mathrm{~cm}^{-3}$, at 270 and $320^{\circ} \mathrm{C}$, respectively) and electrical mobility (from 12.58 to $17.51 \mathrm{~cm}^{2} \mathrm{~V}^{-1} \mathrm{~s}^{-1}$, at 270 and $320^{\circ} \mathrm{C}$, respectively) are consistent with the change of resistivity with the substrate temperature.

From the X-ray diffractograms presented in Fig. 2 and from the results presented in Table 1, for the ZnO:Ga thin films deposited with temperatures in the range of $270-320^{\circ} \mathrm{C}$ (series 1 ), it is possible to observe that all films exhibit a strong (002) diffraction peak at approx. $34.2^{\circ}$, which is relatively close to that of standard $\mathrm{ZnO}$ crystal $\left(34.4^{\circ}\right)$, indicating that the films have a hexagonal wurtzite structure with the [001] preferred orientation [18]. The slight deviation to lower angles of the (002) diffraction from the standard value for undoped $\mathrm{ZnO}$ indicates that tensile lattice strain exists in the film along c-axis direction [13, 19]. According to Bragg's law, the observed reduction of the diffraction angle corresponds to a biaxial in-plane compressive stress in the film, which increases the out-of-plane interplanar spacing, $d[20]$. In a study that involved the effect of substrate temperature (between RT and $230{ }^{\circ} \mathrm{C}$ ) on the properties of Ga-doped ZnO films, J. Park and coworkers [13] reported that the (002) diffraction angle of all the films slightly deviated to lower angles from that of the standard $\mathrm{ZnO}$; they observed that the lattice strain decreased as the deposition temperature increased (and also the angle deviation was smaller), which means that substitution of $\mathrm{Zn}^{2+}$ by $\mathrm{Ga}^{3+}$ is enhanced upon higher deposition temperatures [13]. No other diffraction peaks from other compounds (such as $\mathrm{Ga}_{2} \mathrm{O}_{3}$ ) were registered, indicating that the doped $\mathrm{Ga}$ atoms

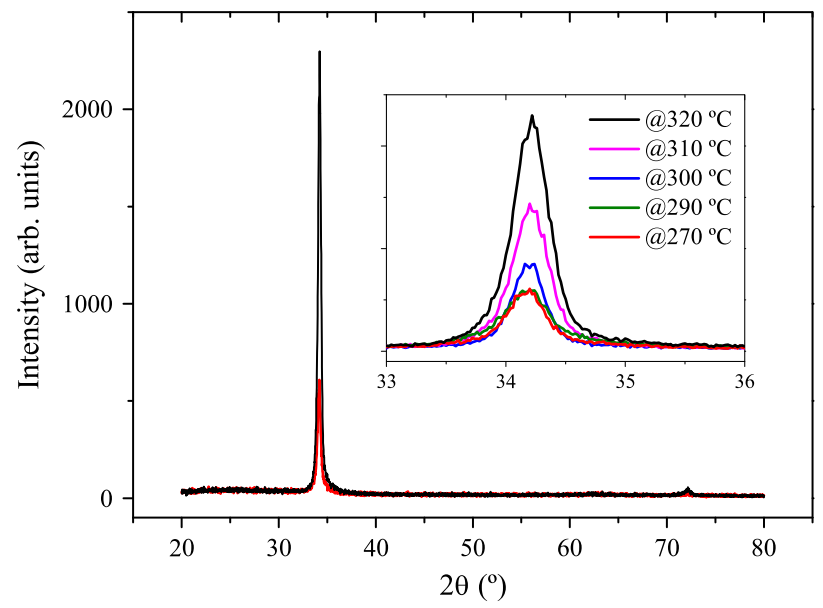

Fig. 2. XRD patterns for $\mathrm{ZnO}$ :Ga films deposited as a function of varyingsubstrate temperatures from 270 to $320^{\circ} \mathrm{C}$, for deposition conditions of series 1 in Table 1. did not change the $\mathrm{ZnO}$ crystal structure, and $\mathrm{Ga}$ atoms replace $\mathrm{Zn}$ in the hexagonal lattice or are segregated to the non-crystalline region in grain boundary $[8,11,21,22]$. As expected, the full width at half maximum of the $\mathrm{ZnO}$ (002) diffraction peak decreases with increasing deposition temperature, indicating an improvement of the crystalline quality [13]. It appears that small crystallites coalesce to make larger crystallites in the ZnO:Ga films [16,23], leading to an increase of the grain size from 22.6 to $42.3 \mathrm{~nm}$, as the substrate temperature slightly increases from 270 to $320{ }^{\circ} \mathrm{C}$ (see Table 1 ). These results corroborate with those obtained from the electrical properties, since a large decrease of electrical resistivity from 270 to $320^{\circ} \mathrm{C}$ is observed. At lower substrate temperature, the phenomenon of migration and diffusion of the sputtering species is inhibited and this definitely seems to suppress the growth of the nuclei. On the other hand, the enhancement of the sputtering species diffusion at higher substrate temperatures leads to the improvement of film crystallinity and increase of the crystallite grain size. This results in a reduced grain boundary scattering, which leads to higher electrical motility (Fig. 1) [13-15].

The optical transmittance spectra of ZnO:Ga films deposited at different substrate temperatures (series 1 in Table 1) are shown in Fig. 3. The average optical transmittance in the visible region $(400-700 \mathrm{~nm}$ ) is $~ 80 \%$ for all films, which also satisfies the requirement for transparent electrode applications $[12,13]$. The high transparency may be associated with a good structural homogeneity and crystallinity [14]. Other authors have also noted that the transmittance (in the visible region) of the films does not change with increasing substrate temperature. S. Wang et al. [14] reported that this may be related to the fact that Ga content in the films has basically become a full oxidation state and, thereby, the increasing of temperature can no longer significantly improve the transmission [14]. The cut-off behavior at the blue end (lower wavelengths) of the optical spectrum is determined by direct electronic transitions from the valence band to the conduction band [8]. The observed blue shift in the absorption edge with the increase of substrate temperature can be associated with the known Burstein-Moss (BM) effect [24]. This blue shift indicates an increase of carrier concentration, which leads to a filling of the small density of states of $\mathrm{ZnO}$ near the conductionband minimum, contributing to the decrease of the electrical resistivity of $\mathrm{ZnO}$ :Ga shown in Fig. 1[8,14,25].

The film thickness, $d$, increases from 0.94 to $1.25 \mu \mathrm{m}$ as the substrate temperature increases from 270 to $320^{\circ} \mathrm{C}$ (see Table 1 ); $d$ was evaluated from the cross section analyses on the SEM micrographs. In a study that involved the effect of substrate temperature (between 50 and $350{ }^{\circ} \mathrm{C}$ ) on the properties of heavy Ga-doped $\mathrm{ZnO}, \mathrm{S}$. Wang and co-workers [14] also observed this trend in the range of substrate temperature between 50 and $250{ }^{\circ} \mathrm{C}$. They reported that probably there is a

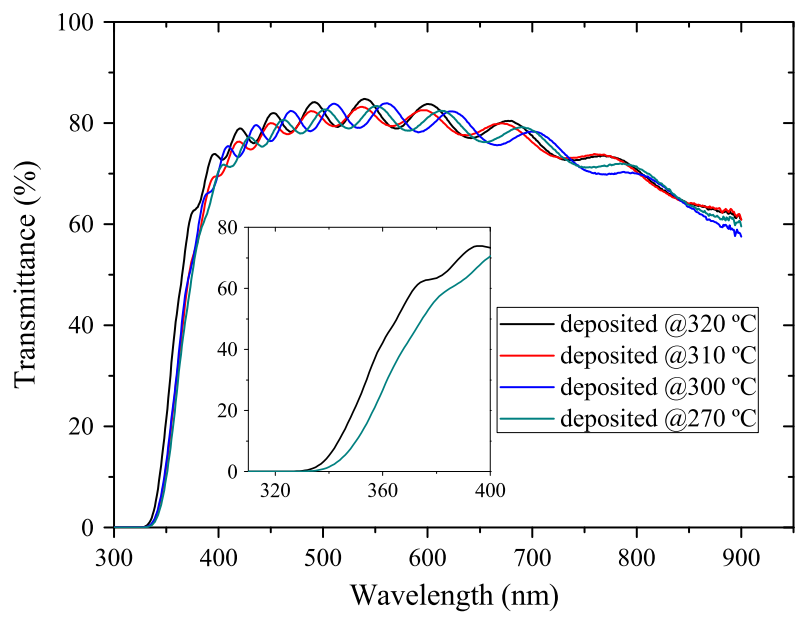

Fig. 3. Effect of substrate temperature on the transmittance spectra of $\mathrm{ZnO}: \mathrm{Ga}$ thin films, for deposition conditions of series 1 in Table 1 . 
reorganization of certain chemical bonds and rupture process happens at the surface of the substrate; therefore, the chemical adsorption is accelerated as the substrate temperature increases, and thus the deposition rate is increased [14].

The SEM micrographs of the cross-section and surface morphology of $\mathrm{ZnO}: \mathrm{Ga}$ films are presented in Fig. 4 for the series 1 samples in Table 1 . There, it is possible to observe that both films deposited at 270 and $320^{\circ} \mathrm{C}$ exhibit vertically aligned columnar structure, which becomes more compact as the substrate temperature increases. At higher substrate temperatures the sputtered particles have more energy to diffuse, resulting in the densification of the film; a higher temperature also favors the c-axis orientation of the growing film $[8,13]$. The film deposited at a substrate temperature of $270{ }^{\circ} \mathrm{C}$ presents more voids, which explains the worst electrical properties obtained at this temperature (this film presents the highest electrical resistivity and the lowest carrier mobility), due to the higher grain boundary scattering. It seems that the number of boundaries that exists between the columns decreases with increasing of temperature, since the grains interact with each other and agglomerate [8]. From the cross-section micrographs, and as shown in Table 1 , it is easily discernible the aforementioned increase of the film thickness as the substrate temperature increases.

\subsection{Effect of sputtering pressure}

The sputtering pressure $\left(p_{s}\right)$ dependence of the electrical properties of ZnO:Ga films is presented in Fig. 5, for samples of series 2 in Table 1. The electrical resistivity of the films is relatively low for an oxide material, and decreases from $1.70 \times 10^{-3} \Omega \cdot \mathrm{cm}$ to $6.81 \times 10^{-4} \Omega \cdot \mathrm{cm}$ as the sputtering pressure increases from 0.30 to $0.42 \mathrm{~Pa}$. The changes of carrier concentration and electrical mobility are consistent with that of electrical resistivity; as the sputtering pressure increases from 0.30 to $0.42 \mathrm{~Pa}$, the carrier concentration increases from $3.39 \times 10^{20}$ to

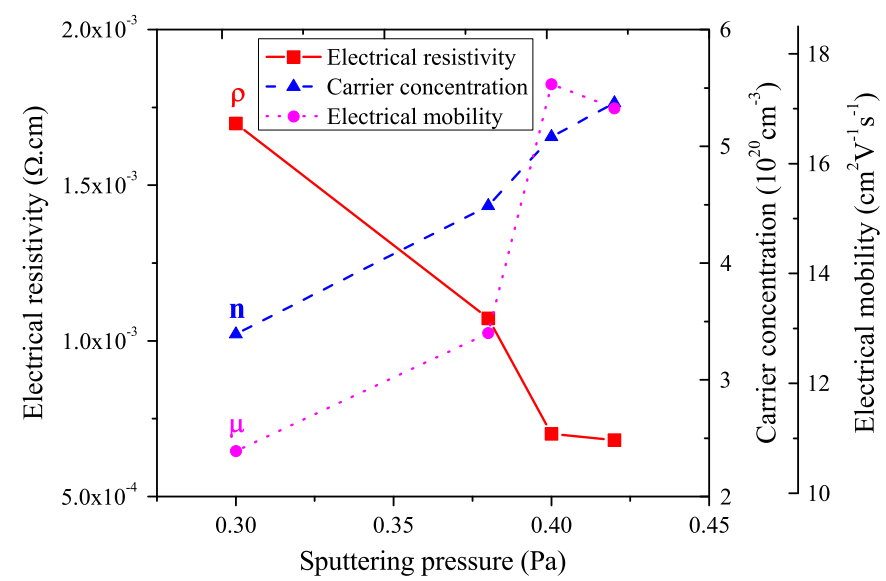

Fig. 5. Effect of different sputtering pressures on the electrical properties of $\mathrm{ZnO}$ :Ga thin films, for deposition conditions of series 2 in Table 1.

$5.37 \times 10^{20} / \mathrm{cm}^{3}$, and electrical mobility increases from 10.82 to $17.07 \mathrm{~cm}^{2} \mathrm{~V}^{-1} \mathrm{~s}^{-1}$.

Fig. 6 shows the XRD patterns of $\mathrm{ZnO}$ :Ga deposited at 0.30, 0.38 and $0.42 \mathrm{~Pa}$ (series 2 in Table 1). All films exhibit a strong [001] preferred orientation [19]. No other diffraction peaks, from other impurity phases, were found. From this figure, it is possible to observe that the intensity of the (002) diffraction peak depends on the sputtering pressure: as the deposition pressure increases, it becomes more intense and sharper; the grain size increases from 13.1 to $53.0 \mathrm{~nm}$ as the sputtering pressure increases from 0.30 to $0.42 \mathrm{~Pa}$ (see Table 1 ). These results also explain the observed decrease of electrical resistivity at higher pressures. The electrical resistivity decreases as the crystallite size increases, since the grain
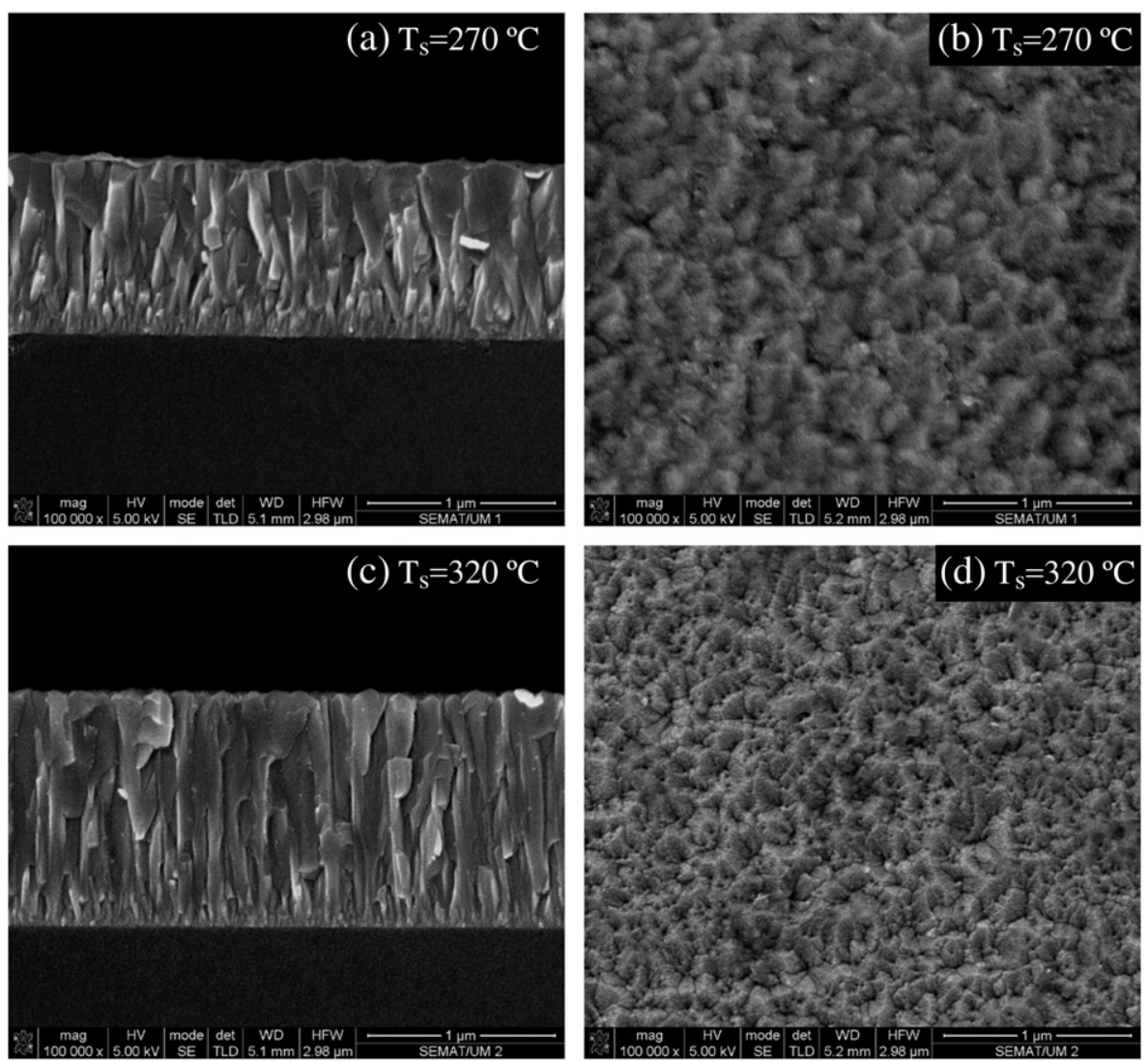

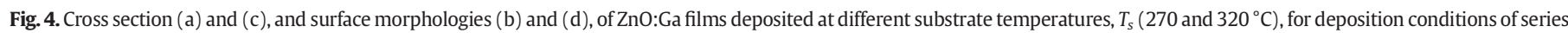
1 in Table 1. 


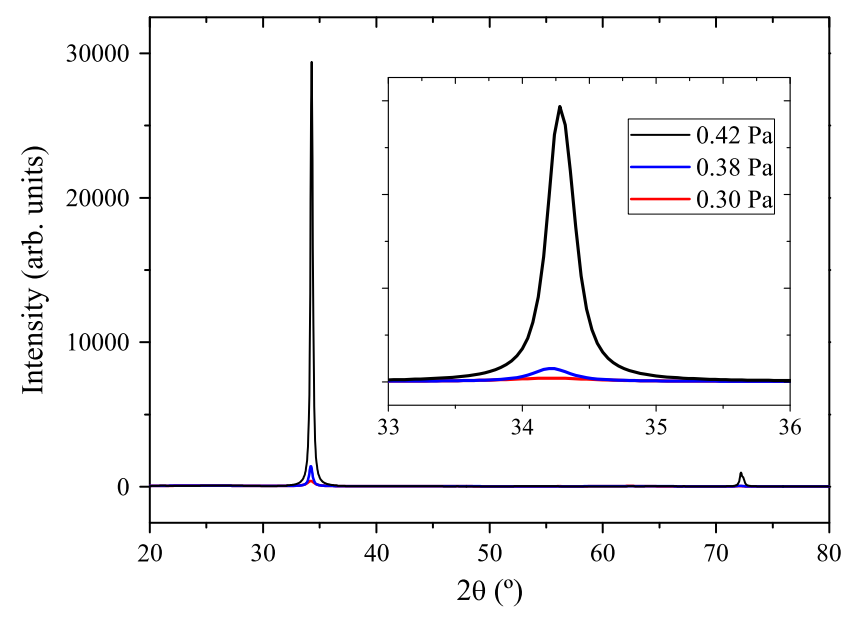

Fig. 6. XRD patterns for $\mathrm{ZnO}$ :Ga films deposited at different sputtering (argon) pressures: $0.30,0.38$ and $0.42 \mathrm{~Pa}$, for deposition conditions of series 2 in Table 1.

boundary acts as a barrier of migration of free carriers [19]. In a study regarding the effect of deposition pressure (from 0.25 to $1.00 \mathrm{~Pa}$ ) on the crystallinity of Al-doped ZnO thin films, Z. Zhang and co-workers [19] observed that the film deposited at $0.50 \mathrm{~Pa}$ (a sputtering pressure close to the highest one in the present study) had the maximum intensity for this diffraction peak. The increase in the sputtering pressure above $0.50 \mathrm{~Pa}$ and concomitant reduction in the energy of the sputtered particles leads to a decrease in the intensity of diffraction peak [19]. On the other hand, at a deposition pressure lower than $0.50 \mathrm{~Pa}$, it was reported a deterioration of the crystallinity quality. In fact, in agreement with the present study, this deterioration is also observed at lower deposition pressure; it seems that at very low deposition pressures the particles when arriving at the substrate surface do not have enough energy (and therefore have low mobility), resulting in more irregular crystalline lattice formation. In another study that involved the influence of different deposition pressures (from 0.133 to $1.463 \mathrm{~Pa}$ ), W. C. Song and co-workers [11] observed that, at pressures between 0.665 and $1.463 \mathrm{~Pa}$, the resistivity of the $\mathrm{ZnO}$ :Ga films increased with the increasing of Ar pressure. However, from 0.133 to $0.665 \mathrm{~Pa}$ (a similar pressure range to that being studied in this manuscript), the resistivity of the ZnO:Ga films decreased with increasing the Ar pressure, achieving a minimum of $1.5 \times 10^{-3} \Omega \cdot \mathrm{cm}$ at a sputtering pressure of $0.665 \mathrm{~Pa}$, which is higher than that obtained in the present study. Based on dispersive X-ray analyses, they observed that with increasing Ar pressure, the $\mathrm{O} / \mathrm{Zn}$ ratio of the $\mathrm{ZnO}$ :Ga films becomes gradually lower at pressures below $0.665 \mathrm{~Pa}$ [11]. In these conditions, the lack of oxygen in the films is relevant, and oxygen vacancies can be created [26]. On the other hand, at higher deposition pressures (above $0.665 \mathrm{~Pa}$ ), the $\mathrm{O} / \mathrm{Zn}$ ratio of the ZnO:Ga films starts to increase with increasing Ar pressure [11]. This suggests that in the present manuscript a very low pressure range is involved, in which the oxygen vacancies are further created with the increasing of argon pressure.

In the present study, the film thickness increases slightly as the sputtering pressure increases from 0.30 to $0.42 \mathrm{~Pa}$ (see Table 1). In a study that involved the effect of deposition pressure (in $\mathrm{Ar}+\mathrm{O}_{2}$ atmosphere), between 0.50 and $2.00 \mathrm{~Pa}$, on the properties of $\mathrm{ZnO}: \mathrm{Ga}$, Q. Ma and co-workers [27] reported that, as the deposition pressure increases, the growth rate increases and reaches a maximum at the deposition pressure of 1.0 Pa, and then decreases with further increase in the deposition pressure. According to Q. Ma and co-workers, lowering deposition pressure (from 1.00 to $0.50 \mathrm{~Pa}$ in their case), leads to larger mean free path of the sputtered particles, which will then have large energies to collide with the substrate, so some of them will be rebounded, which reduces the growth rate of the films and also degrades surface morphology and roughness [27]. This may explain the lower film thickness obtained for lower sputtering pressures on the present study, and also may explain the observed deterioration of the crystallinity at lower pressures, presented in Fig. 6. In their study, they have also observed that the (002) peak diffraction becomes more intense and sharper as the sputtering pressure increases from 0.50 to $1.00 \mathrm{~Pa}$, leading to a concomitant crystallite dimension increase of 21.7 to $27.5 \mathrm{~nm}$ [27]. In the present manuscript it was observed the same trend. The lowest electrical resistivity $\left(6.81 \times 10^{-4} \Omega \cdot \mathrm{cm}\right)$, obtained for the deposition pressure of $0.42, \mathrm{~Pa}$ is very similar to that obtained by $\mathrm{Q}$. Ma et al. $\left(4.48 \times 10^{-4} \Omega \cdot \mathrm{cm}\right.$ obtained for a deposition pressure of $1.00 \mathrm{~Pa}$, which corresponds to a carrier concentration of $1.78 \times 10^{21} / \mathrm{cm}^{3}$ and an electrical mobility of $7.52 \mathrm{~cm}^{2} \mathrm{~V}^{-1} \mathrm{~s}^{-1}$ ). Q. Ma et al. also reported that at lower pressure, the sputtered particles have enough energy to diffuse, resulting in the formation of a more compact film [27]. In fact, from the SEM micrographs of the films, presented in Fig. 7, it is clear that a lower sputtering pressure $(0.30 \mathrm{~Pa})$ leads to larger columns compared to that films deposited at higher sputtering pressure which, in turn, consist in a more porous structure with columns separated by a larger number of voids. From this figure it is also easily discernible that the thickness of the films increases with increasing sputtering pressure, as already reported in Table 1.

The transmittance data for the ZnO:Ga films as a function of the wavelength $(300-900 \mathrm{~nm})$ is shown in Fig. 8 for samples of series 2 in Table 1 . The average transmittance in the visible region is $~ 80 \%$ for all films, and the spectra are similar independently on the deposition pressure. Nevertheless the transmittance in the visible region increases slightly as the sputtering pressure increases from 0.30 to $0.42 \mathrm{~Pa}$. This may be attributed to the higher grain size at higher sputtering pressures [19]. It is not possible to conclude about the influence of the argon pressure on the absorption edge of these $\mathrm{ZnO}$ :Ga films but, it seems, and is also reported by other authors that, a blueshift should be observed as a result of carrier concentration effect for higher pressures.

\subsection{Effect of substrate bias voltage}

Some researchers have already reported that a bias voltage applied to the substrate can attract $\mathrm{Ar}^{+}$cations in the plasma to bombard the growing film. This bombardment will give an additional energy to the molecules and clusters that condense and nucleate on the substrate and peel off the molecules with weak bonding on the growing front of the film [28]. It is believed that this additional energy imputed to the growing film by the bombardment is beneficial for film crystallinity (providing an improvement in film-material density and structure), and could replace the energy provided by a higher substrate temperature [29]. On the other hand, this ion bombardment can induce defects in the film, which influences its electrical properties [30].

The effect of different substrate bias voltages $\left(V_{s}\right)$ on the electrical properties of ZnO:Ga films is illustrated in Fig. 9 (series 3 in Table 1). It is readily observable that lower electrical resistivity values are achieved for lower (absolute) bias voltages; more precisely $3.03 \times 10^{-4} \Omega \cdot \mathrm{cm}$ was reached at a bias voltage of $-40 \mathrm{~V}$. The concomitant increase in electrical resistivity as the absolute bias voltage increases is in agreement with the decrease of carrier concentration and electrical mobility; a carrier concentration and an electrical mobility of $6.99 \times 10^{20} / \mathrm{cm}^{3}$ and $29.49 \mathrm{~cm}^{2} \mathrm{~V}^{-1} \mathrm{~s}^{-1}$, respectively, were obtained at a bias voltage of $-40 \mathrm{~V}$. An increase of the absolute bias voltage up to $-120 \mathrm{~V}$ results in a gradually increase of electrical resistivity to a maximum value of $1.29 \times 10^{-3} \Omega \cdot \mathrm{cm}$, accompanied by a decrease of both carrier concentration and electrical mobility, which may be explained by the excessive re-sputtering of ion bombardment on the substrate surface, smaller crystallite sizes, more grain boundaries and microvoids [31-33].

In Fig. 10 it is presented the XRD patterns for the ZnO:Ga films deposited at different bias voltages (series 3 in Table 1). These results validate those obtained from the electrical measurements in the sense that, in general, the diffraction peak intensity increases as the absolute bias voltage decreases. The most intense peak corresponds to the film 

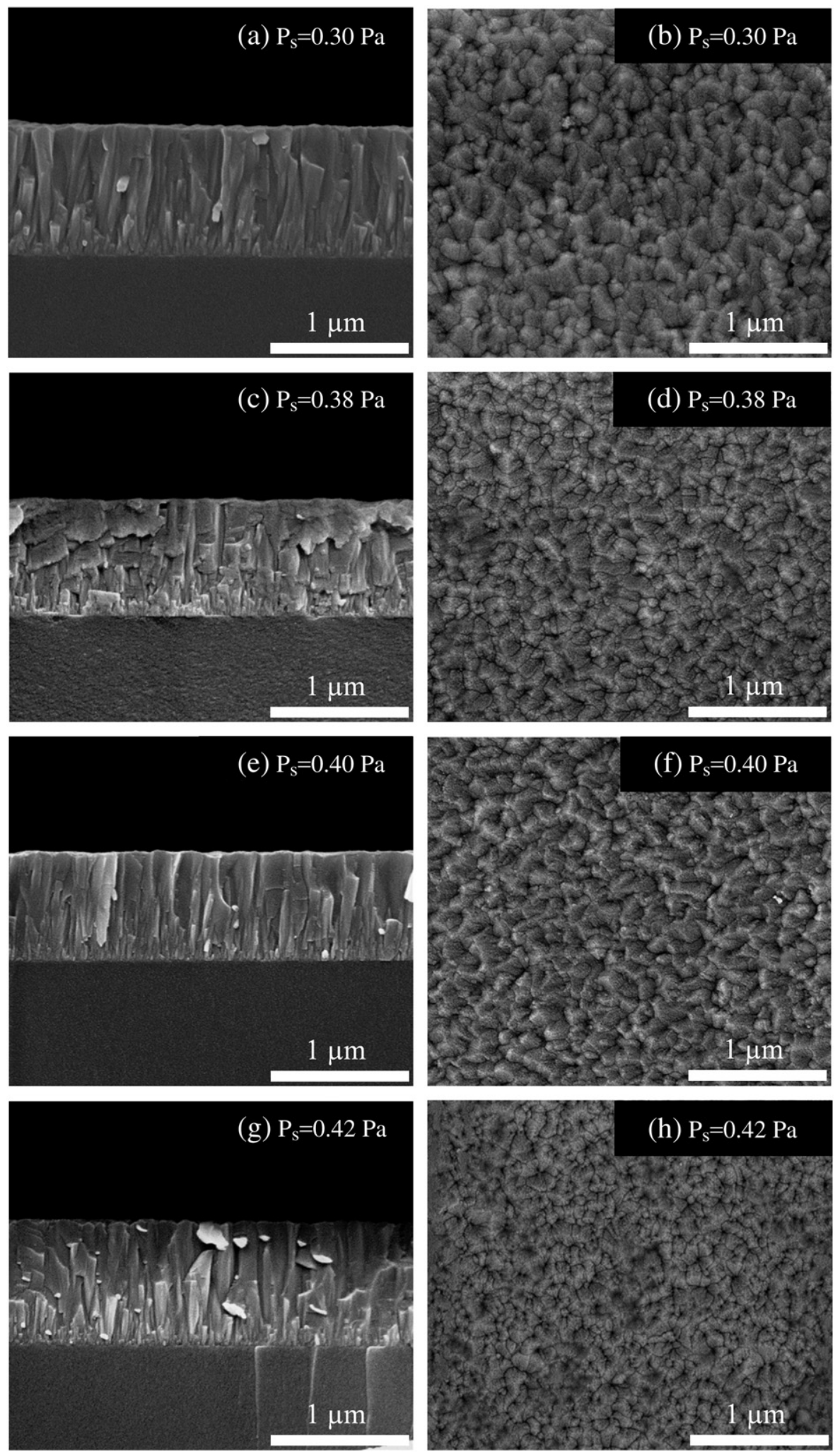

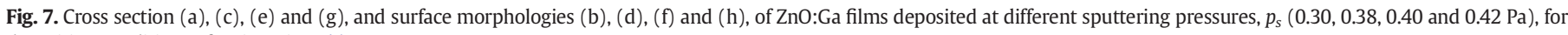
deposition conditions of series 2 in Table 1 .

deposited with a bias voltage of $-40 \mathrm{~V}$, which has also the lowest electrical resistivity (Fig. 1). On the other hand, the less intense peak corresponds to the film deposited at a bias voltage of $-120 \mathrm{~V}$, which has the highest electrical resistivity. A similar behavior, but for $\mathrm{Cu}$ thin films, was also reported by other researchers $[31,32]$.
As seen, the electrical resistivity slightly decreases as the bias voltage varies from 0 (grounded) to $-40 \mathrm{~V}$. According to some researchers, these variations are attributed to improved crystallinity, increased substitutional doping and decreased interstitial atoms, due to the already mentioned bombardment of the substrate caused by the application of 


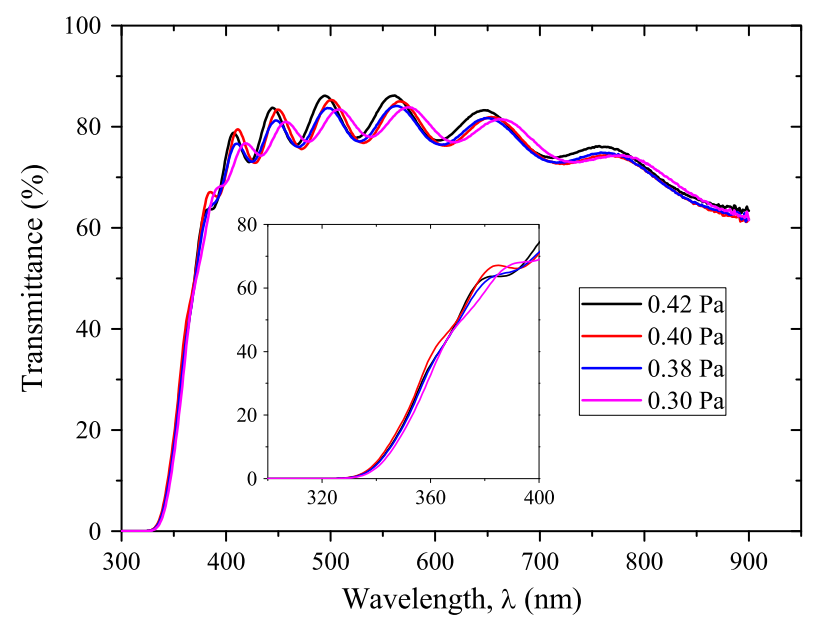

Fig. 8. Effect of different sputtering pressures on the transmittance spectra of $\mathrm{ZnO}$ :Ga thin films, for deposition conditions of series 2 in Table 1.

a negative bias that enhances its temperature [29,34]. In fact, an abrupt improvement of the $\mathrm{ZnO}$ : Ga film crystallinity at a bias voltage of $-40 \mathrm{~V}$ is observed. Moreover, the (002) diffraction angle at a bias voltage of $-40 \mathrm{~V}$ is very similar to that of undoped $\mathrm{ZnO}$, suggesting a better effect of replacement doping. However, as the bias voltage further increases to $-120 \mathrm{~V}$ it is witnessed a decrease of that diffraction peak intensity and an increase of its full width at half-maximum. $\mathrm{H}$. Ma and co-workers [33] attribute these phenomena to the lower deposition rate and smaller crystallite size when higher absolute bias voltages are applied to the substrate, accompanied by an increase of the electrical resistivity. It is also reported that the change of carrier concentration with bias voltage may be explained by the variation of oxygen deficiency in the films. In a study that involved the dependence of the electrical and optical properties on the bias voltage of $\mathrm{ZnO}$ :Al thin films, J. Lee and co-workers [34] observed that, as the absolute bias voltage varies from 0 to $-30 \mathrm{~V}$, the electrical resistivity decreases from $8.5 \times 10^{-4} \Omega \cdot \mathrm{cm}$ to $6.2 \times 10^{-4} \Omega \cdot \mathrm{cm}$; however, when the absolute bias voltage further increased, the electrical resistivity increased. They found that the $\mathrm{Zn} / \mathrm{O}$ ratio changed from 0.89 to 1.09 when the bias voltage changed from grounded to $-30 \mathrm{~V}$ on the substrate. This may explain the minimum electrical resistivity obtained at $-40 \mathrm{~V}$ in the present work. From the film thickness data presented in Table 1, it is possible to see that, initially, the deposition rate increases from 0 to $-40 \mathrm{~V}$, and a maximum film thickness of $1.40 \mu \mathrm{m}$ is obtained. According to $\mathrm{H}$. Ma and co-workers [33], at the low bias voltage range, the increase of the deposition rate is due to the $\mathrm{Ar}^{+}$cation and clusters of the

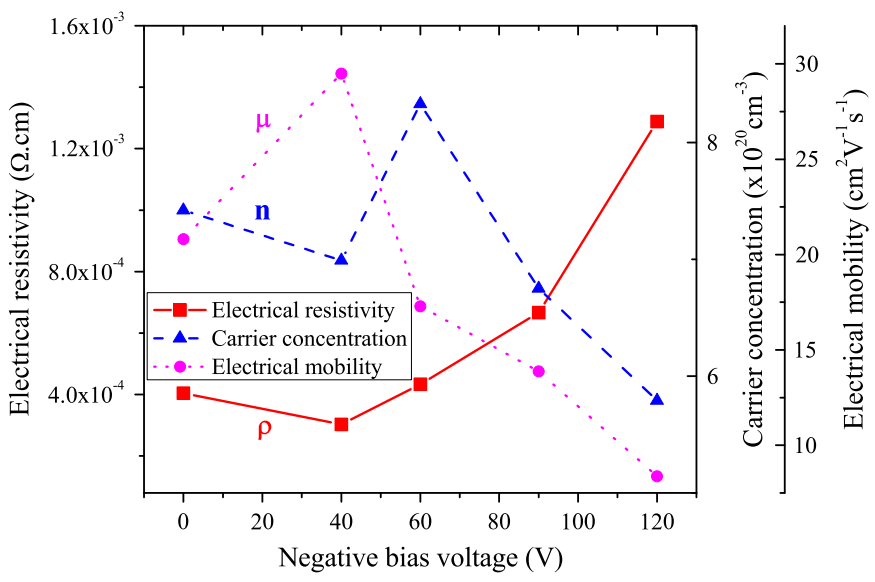

Fig. 9. Substrate bias voltage effect on the electrical properties of $\mathrm{ZnO}$ :Ga thin films, for deposition conditions of series 3 in Table 1.

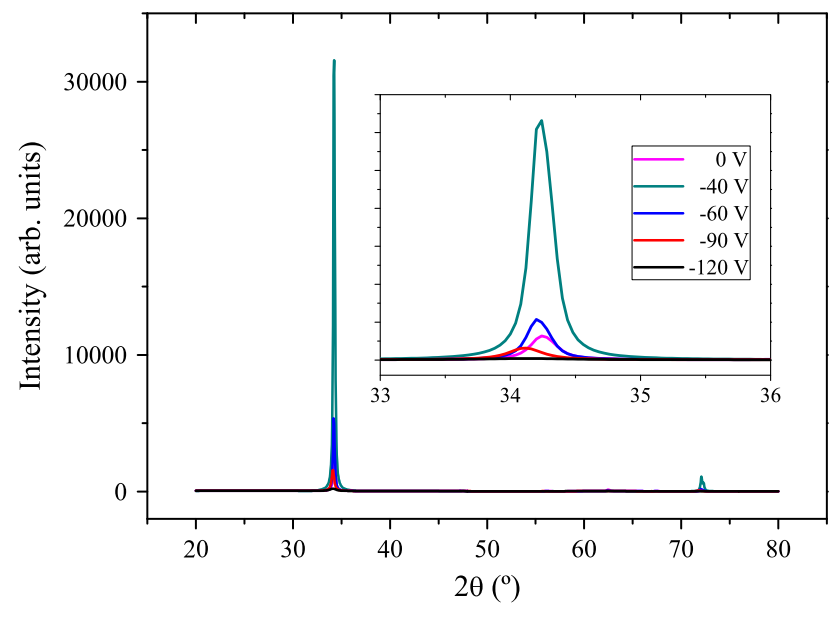

Fig. 10. XRD patterns for $\mathrm{ZnO}$ :Ga films deposited with different substrate bias voltages, for deposition conditions of series 3 in Table 1 .

sputtered material in the plasma being attracted by the substrate, leading to a higher deposition rate and larger crystallite sizes (as also observed in Table 1 ); however, above $-40 \mathrm{~V}$, the deposition rate decreases due to resputtering on the film growing front [33].

Fig. 11 shows the optical transmittance of $\mathrm{ZnO}$ :Ga films as a function of negative bias voltage (series 3 in Table 1 ). The average transmittance in the visible region is $\sim 80 \%$ for all applied bias voltages. It is easily observable that the decrease of the absolute bias voltage leads to a shift of the absorption edge to shorter wavelengths, as expected from the electrical property results presented in Fig. 9, indicating an increase of the optical band gap [28,34]. In a study that involved the influence of bias voltage on the $\mathrm{ZnO}$ :Al properties, $\mathrm{H}$. Ma and co-workers [33] observed the same trend. They reported that as the absolute bias voltage increases, the crystallite size increases leading to less grain boundary and microvoids and, thereby, the carrier concentration of the samples increased because of more clearly effect of replacement doping [33]. From Table 1 it is possible to observe the inverse relationship regarding the grain size increase with the absolute bias voltage decrease. The widening of band-gap due to the increasing of carrier concentration is attributed to the aforementioned Burstein-Moss shift.

The morphologies of $\mathrm{ZnO}$ : Ga films as a function of the bias voltage are presented in Fig. 12, for samples of series 3 from Table 1. As the absolute bias voltage increases from 0 to $-40 \mathrm{~V}$, the grain size increases from 55.7 to $74.3 \mathrm{~nm}$ (see Table 1), leading to a decrease in the ratio of grain boundaries, due to the additional energy that enhances the densification of the film microstructure. However, when the absolute bias

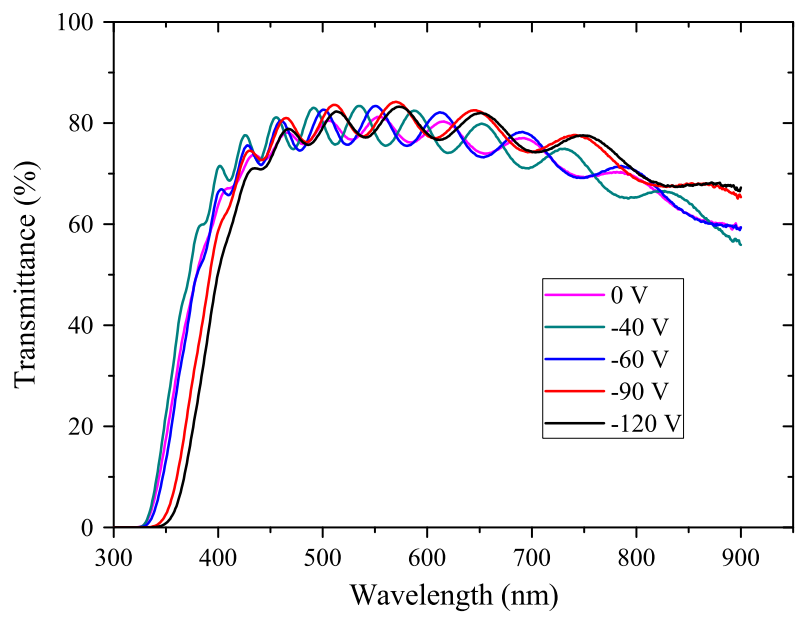

Fig. 11. Substrate bias voltage effect on the transmittance spectra of $\mathrm{ZnO}$ :Ga thin films, for deposition conditions of series 3 in Table 1. 

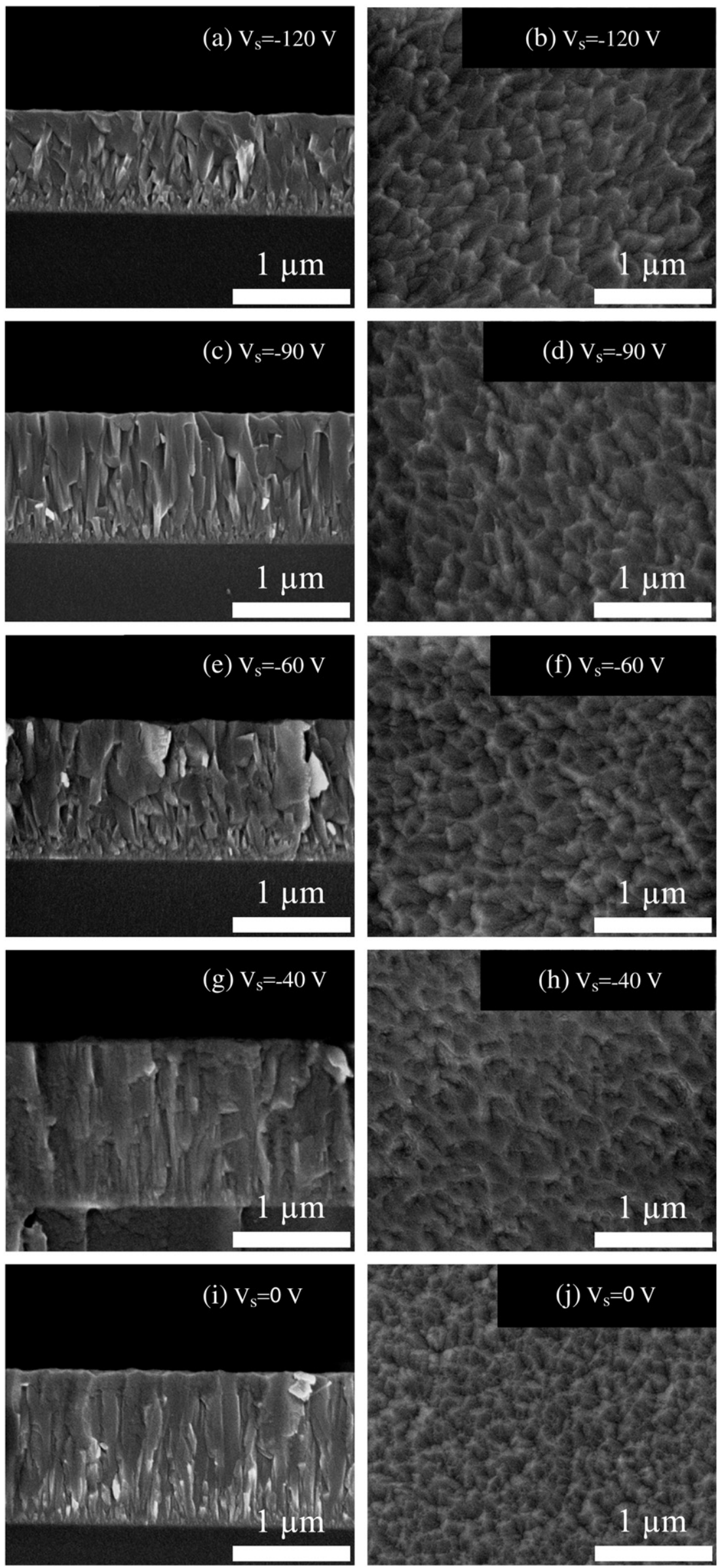
voltage is higher than $-40 \mathrm{~V}$, higher ion bombarding on the growing film takes place, resulting in a decrease in density and, conversely, an increase of the number of voids presented in the film. As the negative bias voltage is varied from $-40 \mathrm{~V}$ to $-120 \mathrm{~V}$ the crystallite size is reduced from $74.3 \mathrm{~nm}$ to $12.7 \mathrm{~nm}$. This peening effect, which is a characteristic of enhanced ionic bombardment on the film growing front, acts as a microstructural refinement and impacts in the form of an inhibition of crystalline grain growth. These results support those obtained from the electrical measurements and are in agreement with that obtained from the XRD analyses.

\section{Conclusion}

Highly transparent and conductive ZnO:Ga films (with a thickness of $\sim 1 \mu \mathrm{m}$ ) were deposited on glass surfaces, by d.c. pulsed magnetron sputtering from a GZO ceramic target, in an argon ( $\mathrm{Ar}$ ) atmosphere. The influence of different process parameters: substrate temperature, sputtering pressure and bias voltage on the electrical, optical, structural and morphological properties was experimentally investigated in detail. All ZnO:Ga films have an average transmittance of $~ 80 \%$ in the visible region and present a preferential crystalline orientation along the [001] direction, regardless of the deposition conditions. It was found that a higher deposition temperature led to lower electrical resistivity. At higher temperatures, more Ga atoms are activated into the $\mathrm{ZnO}$ lattice as donors (there is an increase in diffusion of $\mathrm{Ga}$ atoms from interstitial locations and grain boundaries into the $\mathrm{Zn}$ cation sites), leading to an increase in carrier concentration, since the Ga atom is trivalent and $\mathrm{Zn}$ has a valence of 2 . Furthermore, there is an improvement of the crystalline quality at higher substrate temperatures. A higher sputtering pressure (within the studied range) proved to be favorable in obtaining lower electrical resistivity. This may be related to the fact that lower sputtering pressure leads to larger mean free path of the sputtered particles, which will then have large energies to collide with the substrate, so some of them will be rebounded. This leads to the deterioration of the crystallinity, with which the increase of electrical resistivity is also associated. Finally, it was shown that a low bias voltage, more precisely, $-40 \mathrm{~V}$, abetted in obtaining the lowest electrical resistivity of $3.03 \times 10^{-4} \Omega \cdot \mathrm{cm}$, due to the additional energy imputed to the growing film by the bombardment of $\mathrm{Ar}^{+}$ions to the growing film, which is also beneficial to the improvement of the crystallinity of the film. On the other hand, an increase of the absolute bias voltage above the latter value led to a deterioration of the electrical properties, associated with the excess of ion bombardment induced defects. These properties that were attained for the ZnO:Ga films fulfill the necessary requirements for their application as transparent electrodes in photovoltaic cells.

\section{Acknowledgments}

The authors acknowledge the funding from the Portuguese Innovation Agency (AdI), project reference WinDSC-21539, co-funded by FEDER/POFC.

\section{References}

[1] K. Lai, C. Liu, C. Lu, C. Yeh, M. Houng, Characterization of ZnO:Ga transparent contact electrodes for microcrystalline silicon thin film solar cells, Sol. Energy Mater. Sol. Cells 94 (2010) 397.

[2] Q. Ma, Z. Ye, H. He, J. Wang, L. Zhu, B. Zhao, Preparation and characterization of transparent conductive $\mathrm{ZnO}: G a$ films by DC reactive magnetron sputtering, Mater. Charact. 59 (2008) 124.

[3] V. Assunção, E. Fortunato, A. Marques, H. Águas, I. Ferreira, M.E.V. Costa, R. Martins, Influence of the deposition pressure on the properties of transparent and conductive $\mathrm{ZnO}$ :Ga thin-film produced by r.f. sputtering at room temperature, Thin Solid Films 427 (2003) 401.

[4] J. Nomoto, M. Konagai, K. Okada, T. Ito, T. Miyata, T. Minami, Comparative study of resistivity characteristics between transparent conducting AZO and GZO thin films for use at high temperatures, Thin Solid Films 518 (2010) 2937.
[5] H.J. Ko, Y.F. Chen, S.K. Hong, H. Wenisch, T. Yao, D.C. Look, Ga-doped ZnO films grown on GaN templates by plasma-assisted molecular-beam epitaxy, Appl. Phys. Lett. 77 (2000) 3761.

[6] S. Choopun, R.D. Vispute, W. Noch, A. Balsamo, R.P. Sharma, T. Venkatesan, A. Iliadis, D.C. Look, Oxygen pressure-tuned epitaxy and optoelectronic properties of laserdeposited ZnO films on sapphire, Appl. Phys. Lett. 75 (1999) 3947.

[7] Q. Ma, Z. Ye, H. He, L. Zhu, J. Wang, B. Zhao, Influence of $\mathrm{Ar} / \mathrm{O}_{2}$ ratio on the properties of transparent conductive $\mathrm{ZnO}: \mathrm{Ga}$ films prepared by $\mathrm{DC}$ reactive magnetron sputtering, Mater. Lett. 61 (2007) 2460.

[8] X. Bie, J.G. Lu, L. Gong, L. Lin, B.H. Zhao, Z.Z. Ye, Transparent conductive ZnO:Ga films prepared by DC reactive magnetron sputtering at low temperature, Appl. Surf. Sci. 256 (2009) 289.

[9] P.J. Kelly, Y. Zhou, A. Postill, A novel technique for the deposition of aluminumdoped zinc oxide films, Thin Solid Films 426 (2003) 111.

[10] C.J. Tavares, M.V. Castro, E.S. Marins, A.P. Samantilleke, S. Ferdov, L. Rebouta, M. Benelmekki, M.F. Cerqueira, P. Alpuim, E. Xuriguera, J.-P. Rivière, D. Eyidi, M.-F. Beaufort, A. Mendes, Effect of hot-filament annealing in a hydrogen atmosphere on the electrical and structural properties of $\mathrm{Nb}$-doped $\mathrm{TiO}_{2}$ sputtered thin films, Thin Solid Films 520 (2012) 2514.

[11] W.C. Song, S.I. Kwon, G.S. Kang, J.H. Park, K.J. Yang, D.G. Lim, Characteristics of GZO films prepared by using a rf magnetron plasma at low temperature, J. Korean Phys. Soc. 53 (2008) 2522

[12] K. Lee, E.K. Kim, H. Kim, H. Kang, J. Song, Low temperature Al doped ZnO films on a flexible substrate by DC sputtering, Phys. Status. Solidi C 5 (2008) 3344.

[13] J. Park, B. Shin, H. Moon, M. Lee, K. Park, K. Ahn, W. Lee, J. Myoung, Effect of the substrate temperature on the properties of Ga-doped $\mathrm{ZnO}$ films for photovoltaic cell applications deposited by a pulsed DC magnetron sputtering with a rotating cylindrical target, Vacuum 86 (2012) 1423.

[14] S. Wang, X. Li, J. Zhang, Effects of substrate temperature on the properties of heavy Ga-doped ZnO transparent conductive film by RF magnetron sputtering, J. Phys. Conf. Ser. 188 (2009) 012017.

[15] H. Kim, C.M. Gilmore, A. Piqué, J.S. Horwitz, H. Mattoussi, H. Murata, Z.H. Kafafi, D.B. Chrisey, Electrical, optical, and structural properties of indium tin-oxide thin films for organic light-emitting devices, J. Appl. Phys. 86 (1999) 6451.

[16] S. Park, T. Ikegami, K. Ebihara, Effects of substrate temperature on the properties of Ga-doped ZnO by pulsed laser deposition, Thin Solid Films 513 (2006) 90.

[17] G.A. Hirata, J. McKittrick, J. Siqueiros, O.A. Lopez, T. Cheeks, O. Contreras, J.Y. Yi, High transmittance-low resistivity ZnO:Ga films by laser ablation, J. Vac. Sci. Technol. A 14 (1996) 791.

[18] ICDD card number 01-089-1397.

[19] Z. Zhang, C. Bao, S. Ma, L. Zhang, S. Hou, Effects of deposition power and pressure on the crystallinity of Al-doped $\mathrm{ZnO}$ thin films at glass substrates by low temperature RF magnetron sputtering, J. Aust. Ceram. Soc. 48 (2012) 214.

[20] K.S. Shtereva, V. Tvarozek, I. Novotny, P. Sutta, M. Milosavljevic, A. Vincze, M. Vojs, S. Flickyngerova, Effect of annealing on properties of sputtered and nitrogenimplanted ZnO:Ga thin films, EPJ Phovolt. 3 (2012) 35003-p1.

[21] X. Yu, J. Ma, F. Ji, Y. Wang, X. Zhang, C. Cheng, H. Ma, Preparation and properties of ZnO:Ga films prepared by r.f. magnetron sputtering at low temperature, Appl. Surf. Sci. 239 (2005) 222.

[22] J.S. Zhang, H.D. Yang, B. Huang, S. Yu, L.X. Zeng, Dependence of argon gas pressure on the structure and photoelectric of Ga doped ZnO thin films deposited by DC magnetron sputtering, Symp. on Photonics and Optoelectronics (SOPO) 2012, p. 1.

[23] V. Gupta, A. Mansingh, Influence of postdeposition annealing on the structural and optical properties of sputtered zinc oxide film, J. Appl. Phys. 80 (1996) 1063.

[24] E. Burstein, Anomalous optical absorption limit in InSb, Phys. Rev. 93 (1954) 632.

[25] K. Kim, Y. Park, Large and abrupt optical band gap variation in In-doped ZnO, Appl. Phys. Lett. 78 (2001) 475.

[26] B. Huang, D. Zhu, X. Ma, Great influence of the oxygen vacancies on the ferromagnetism in the Co-doped ZnO films, Appl. Surf. Sci. 253 (2007) 6892.

[27] Q. Ma, Z. Ye, H. He, L. Zhu, B. Zhao, Effects of deposition pressure on the properties of transparent conductive $\mathrm{ZnO}: \mathrm{Ga}$ films prepared by DC reactive magnetron sputtering, Mater. Sci. Semicond. Process. 10 (2007) 167.

[28] Z.W. Yang, S.H. Han, T.L. Yang, L. Ye, D.H. Zhang, H.L. Ma, C.F. Cheng, Bias voltage dependence of properties for depositing transparent conducting ITO films on flexible substrate, Thin Solid Films 366 (2000) 4.

[29] N. Danson, I. Safi, G.W. Hall, R.P. Howson, Techniques for the sputtering of optimum indium-tin oxide films on to room-temperature substrates, Surf. Coat. Technol. 99 (1998) 147.

[30] K. Shtereva, V. Tvarozek, P. Sutta, J. Kovac, I. Novotny, Experimental studies on doped and Co-doped ZnO thin films prepared by RF diode sputtering, in: K. Takahata (Ed.), Micro Electronic and Mechanical Systems, InTech, Olajnica - Vukovar, Croatia 2009, p. 211.

[31] J. Lim, Y. Ishikawa, K. Miyake, M. Yamashita, M. Isshiki, Influence of substrate bias voltage on the properties of $\mathrm{Cu}$ thin films by sputter type ion beam deposition, Mater. Trans. 43 (2002) 1403.

[32] J. Lim, M. Isshiki, Effect of substrate bias voltage on the texture and microstructure of Cu thin films deposited by ion beam deposition, Met. Mater. Int. 9 (2003) 201.

[33] H. Ma, X. Hao, J. Ma, Y. Yang, S. Huang, F. Chen, Q. Wang, D. Zhang, Bias voltage dependence of properties for ZnO:Al films deposited on flexible substrate, Surf. Coat. Technol. 161 (2002) 58.

[34] J. Lee, J. Song, Dependence of the electrical and optical properties on the bias voltage for ZnO:Al films deposited by r.f. magnetron sputtering, Thin Solid Films 516 (2008) 1377.

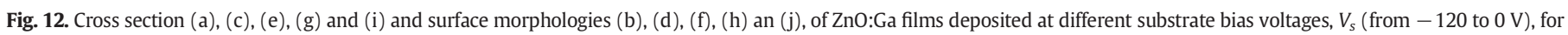
deposition conditions of series 3 in Table 1. 